\title{
Using Authentic Medication Errors to Promote Pharmacy Student Critical Thinking and Active Learning
}

Reza Karimi, RPh, PhD; Jeff Fortner, PharmD; Deepa Rao, PhD; and Joe Su, PhD

Pacific University Oregon School of Pharmacy

\begin{abstract}
Objective: To promote first year (P1) pharmacy students' awareness of medication error prevention and to support student learning in biomedical and pharmaceutical sciences. Innovation: A novel curricular activity was created and referred to as "Medication Errors and Sciences Applications (MESA)". The MESA activity encouraged discussions of patient safety among students and faculty to link medication errors to biomedical and pharmaceutical sciences, which ultimately reinforced student learning in P1 curricular topics.

Critical Analysis: Three P1 cohorts implemented the MESA activity and approximately 75\% of students from each cohort completed a reliable assessment instrument. Each $\mathrm{P} 1$ cohort had at least 14 student teams who generated professional reports analyzing authentic medication errors. The quantitative assessment results indicated that $70-85 \%$ of students believed that the MESA activity improved student learning in biomedical and pharmaceutical sciences. More than $95 \%$ of students agreed that the MESA activity introduced them to medication errors. Approximately $90 \%$ of students agreed that the MESA activity integrated the knowledge and skills they developed through the P1 curriculum, promoted active learning and critical thinking, and encouraged students to be self-directed learners. Furthermore, our data indicated that approximately $90 \%$ of students stated that the achievement of Bloom's taxonomy's six learning objectives was promoted by completing the MESA activity. Next Steps: Pharmacy students' awareness of medication errors is a critical component of pharmacy education, which pharmacy educators can integrate with biomedical and pharmaceutical sciences to enhance student learning in the P1 year.
\end{abstract}

Keywords: medication errors, student learning, P1 curriculum

\section{Description of Problem}

Health professions licensees who work with prescription and non-prescription drugs, i.e., pharmacists and prescribing physicians, optometrists, physician assistants, dentists, and nurses are prone to medication errors in fulfilling their daily responsibilities. A study that assessed the importance of teaching medication errors to medical students demonstrated that this increased students' confidence to communicate medical errors to their patients. ${ }^{1}$ In another study conducted by Kiersma et al., it was shown that implementing active learning in the area of medication errors, in a first year (P1) pharmacy laboratory setting, enhanced student learning in recognizing the importance of patient safety. ${ }^{2}$ Accordingly, teaching the above health professions students, particularly pharmacy students, to identify and prevent medication errors in various patient care settings is anticipated to reduce adverse events associated with prescription and non-prescription drugs.

The Accreditation Council for Pharmacy Education (ACPE) recognizes the importance of educating pharmacy students

Corresponding author: Reza Karimi, RPh, PhD

Dean and Professor

Pacific University School of Pharmacy

222 SE 8th Avenue, HPC-Ste 451, Hillsboro, OR 97123

Phone: 503.352.7276; fax: 503.352.7270

Email: karimir@pacificu.edu about patient safety and the prevention of medication errors (Standards 2016, Appendix 1). ${ }^{3}$ In addition, The North American Pharmacist Licensure Examination (NAPLEX) blueprint indicates the importance of graduating students' skill to evaluate and respond to medication errors. ${ }^{4}$ Furthermore, domain 2 of the 2013 Center for the Advancement of Pharmacy Education (CAPE) Outcomes has identified promoting medication safety in patient care as an important component that entry-level graduates should possess. ${ }^{5}$ Similarly, the recently developed Entrustable Professional Activities (EPAs) domain 5 emphasizes pharmacy graduates should be able to identify underlying system-associated causes of error. ${ }^{6}$

Despite the above guidelines, it is not clear to what extent colleges and schools of pharmacy curricula deliver the craft of understanding medication errors and patient safety to their students. At Pacific University School of Pharmacy, we have designed a curricular activity to acquaint students with some of the most egregious national and international medication errors that have caused devastating consequences. This activity has assisted students in identifying root causes for reported medication errors and in discussing manageable plans to promote medication safety. This article describes a method for educating pharmacy students in real-world medication errors and utilizing the lessons from these errors to assist students in learning biomedical and pharmaceutical sciences. The entire curricular activity for each cohort was implemented within 3 days ( 2 hours/day). 


\section{Statement of the Innovation}

It is important to introduce pharmacy students to real-world medication errors and the devastating impact errors bring about to patients and their family members or caregivers. Equally, it is imperative to educate students on what steps one must take to mitigate medication errors in different practice settings. We have designed an innovative curricular activity to introduce students to some of the most devastating national and international medication errors. Further, using these errors, we have created an environment to assist students in learning biomedical and pharmaceutical science concepts.

\section{The Educational Innovation}

With ever-increasing access to the media, examples of authentic medication errors are readily available to the public, including pharmacy students. Two major objectives of this study were to: 1) use authentic medication errors to direct student attention to the significant impacts that errors have on patients, patient caregivers, healthcare providers, and the healthcare community at large and 2) employ the first objective to enhance student learning in biomedical and pharmaceutical sciences during the P1 year by promoting critical thinking, active learning, and self-directed learning. To implement both objectives, a team, including two pharmacists and three pharmaceutical sciences faculty, generated a novel learning tool referred to as "Medication Errors and Sciences Applications" (MESA). Student teams were asked to generate a MESA report that included information about a specific and authentic medication error related to a specific drug product that faculty identified on public media (i.e. a total of at least 14 medication errors/cohort). Examples of medication errors that caused devastative effects and were discussed among student teams included colchicine, $\mathrm{NaCl}, \mathrm{CaCl}$, arginine, epinephrine, prednisone, heparin, synthroid, Biodyl (a veterinary case), and the well-known outbreak of meningitis caused by contaminated steroid injections. Three P1 cohorts (classes of 2015, 2016, and 2018), (approximately 95 students/cohort), implemented the MESA activity. We built at least 14 student teams (6-7 students/team) per cohort. Student teams from cohorts 1 (class of 2015) and cohort 2 (class of 2016) were asked to generate a 2-3 page MESA report and PowerPoint presentations over at least two 2-hour in-class sessions. Cohort 3 (class of 2018) had 16 student teams. Eight student teams were asked to generate a 2-3 page MESA report and the other eight student teams were asked to generate their reports in the form of PowerPoint presentations over at least two 2-hour in-class sessions. All three cohorts were required to follow a consistent 3-day step-by-step process to generate their reports. This process included day 1: assembling a student team, reviewing a given authentic medication error, discussing the medication error and following instructions given in Table 1 to draft a MESA report with a reference list; day 2: bridging their medication error with biomedical and pharmaceutical sciences, completing all the required assignments indicated in Table 1 and finalizing the MESA report and/or PowerPoint presentations; and day 3: presenting a 15-minute presentation and discussing their findings from the medication error with their peers and faculty. Based on faculty discussion with students, we realized that some student teams encountered difficulty in bridging a medication error with certain subject areas. For instance, two teams found it difficult to connect a high dose of $\mathrm{CaCl}$ to medicinal chemistry. As a result, it is prudent to identify medication errors that can readily be linked to pharmaceutical and biomedical sciences. For each cohort presentation, we provided 1.5 hours of Oregon Board of Pharmacy approved Continuing Education (CE) in medication safety. During the presentation day, a small number of faculty, beyond the MESA instructors, attended student presentations to receive CE credits. After completion of all presentations, MESA instructors emphasized the importance of patient safety to all teams and provided feedback to improve students' presentation skills.

Table 1 summarizes a series of questions that student teams were required to address in order to implement their MESA activity and generate their reports. Student teams were asked to integrate biochemistry, medicinal chemistry, pharmacology, pharmacokinetics, pharmaceutics, and drug information topics to the selected drug product and its medication error. In addition, student teams were asked to write at least two take home messages ("Essential Keys"). An essential key was an important pharmaceutical, biomedical, clinical science, or pharmacy related concept specifically tailored to the medication error. Each P1 student was asked to recognize, know, answer, and discuss upon presentation a particular essential key. A few essential keys that were provided by the student teams include: the importance of patient counseling and patient education, review of medication history, being mindful of drugs that sound/look alike, following the United States Pharmacopeia (USP) guidelines when preparing a sterile solution, establishing clear process and communication to mitigate and report medication errors, and educating pharmacists' staff of potential medication errors that may occur at their pharmacy sites. Student teams were encouraged to access reliable resources, such as package inserts, our online library, textbooks, the Institute for Safe Medication Practices (ISMP), and lecture notes to complete their MESA reports.

\section{Critical Analysis}

In order to assess the effectiveness of each MESA activity and identify areas for improvement, a survey tool was implemented with questions generated based on past published survey questions. ${ }^{7}$ Each survey question was explained in the survey instrument to make sure that we gathered accurate and reliable information. For instance, active learning was defined as "an active learning process where the student utilizes and refers to his/her own knowledge to answer a question and also actively seeks and explores other resources to gather relevant information to 
improve or find a better answer". ${ }^{7}$ Similarly, critical-thinking skills were defined as "the intellectual skills to critically interpret and evaluate a concept or a problem in order to synthesize or find an accurate answer to a question". ${ }^{7}$ Despite the fact that the completion of the surveys was not mandatory, approximately 220 students ( $\approx 75 \%$ of each cohort) completed the surveys.

Approximately, $70 \%$ of students agreed that the MESA activity assisted them in increasing their knowledge base in medicinal chemistry, biochemistry, and pharmaceutics. Higher agreements (75-85\%) are seen with pharmacokinetics, pharmacology, drug information, literature searches, and professional presentations. These data were gathered from two P1 cohorts. Table 2 indicates student perceptions of the effectiveness of the MESA activity in assisting students in promoting their knowledge or skills. These data were gathered from the above three P1 cohorts. The MESA activity was also designed to promote teamwork. Our assessment data indicated that a large number of students believed the MESA activity improved their teamwork dynamic, and that patient education and generation of a medication safety plan could reduce medication errors. In addition, our data indicated that the MESA activity promoted student critical thinking, active learning, and self-directed learning. Furthermore, all three classes believed that the MESA activity was an innovative learning tool and that it assisted them in applying integrative pharmaceutical sciences to patient care. When we used Fink's taxonomy, ${ }^{8}$ similarly, a significant number of students believed the MESA activity fostered caring (Table 2).

We were interested in knowing whether the MESA activity incorporated various levels of learning as indicated in Bloom's taxonomy. Bloom's taxonomy is comprised of six learning objectives of increasing complexity, which are Knowledge, Comprehension, Application, Analysis, Synthesis, and Evaluation. ${ }^{9 \& 10}$ In order to gather accurate data, we directly incorporated descriptions of Bloom's taxonomy into our survey instrument. As Table 3 indicates, all learning levels were highly rated, with the lowest rating $(88.7 \% \pm 3.92)$ in Evaluation and the highest in Analysis and Application domains (94.6\% \pm 1.55).

In the same survey instruments, all three cohorts were asked to identify the most significant learning experience from the entire MESA project. The qualitative data indicated that the awareness of medication errors alone or a combination of awareness and prevention of medication errors were highly selected by all three cohorts. A small percentage of students indicated teamwork, presentations, and integration of different biomedical and pharmaceutical sciences as the most significant learning experience that they gained from the MESA activity.

\section{Key Findings}

The MESA activity is built with multifaceted themes to integrate biomedical and pharmaceutical sciences and connect them to medication errors to promote student learning in the P1 curriculum. Our survey results indicated that the MESA activity produced the following outcomes:

1. Reinforcement of knowledge delivered in the $\mathrm{P} 1$ curriculum (Table 2). The integration of medication errors to biomedical and pharmaceutical sciences, and presenting their MESA report, promoted and reinforced student learning in P1 curricular topics.

2. An increased awareness of medication errors and patient education in reducing medication errors (Table 2). We hypothesized that this innovative activity helped students to care and appreciate their role in promoting medication safety and preventing medication errors. Our hypothesis was confirmed when we reviewed the submitted qualitative "Essential Key" from each team. Additionally, the use of Fink's taxonomy assisted us in confirming the above hypothesis by assessing students' engagement and excitement.

3. Utilization of critical thinking, active learning, and self-directed learning (Table 2). Questions presented in Table 1 encouraged students to critically review and evaluate the medication errors and collaborate with each other to actively engage in addressing problems and finding answers to the questions. This process encouraged students to be self-directed learners by using their knowledge and resources from the P1 curriculum.

\section{Next Steps}

Pharmacy students' awareness of medication errors and error prevention is a critical component of pharmacy education and patient care. A structured activity to review authentic medication error cases assists students in becoming aware of the impact patient safety has not only on patients, but also on healthcare providers and the healthcare system at large. Linking medication errors to biomedical and pharmaceutical sciences not only promotes, but also reinforces student learning in P1 curricular topics. Further, sharing this knowledge among peers improves student presentation skills. We have expanded the MESA activity to exclusively link medication errors to pharmaceutics and the craft of compounding, and our preliminary results indicate a positive student learning outcome. We encourage pharmacy faculty to consider generating innovative curricular activities to draw student attention to medication errors and increase student learning in the prevention of medication errors. 


\section{Acknowledgments}

We sincerely appreciate Pacific University School of Pharmacy P1 students from Classes of 2015, 2016, and 2018 who diligently participated in this project. In addition, we extend our earnest appreciation to our colleagues Drs. Amber Buhler and Brigg Turner who provided valuable comments about this study, and Drs. Kristen Malabanan and Aleksandar Todorovic who facilitated the implementation of this project for the class of 2016 cohort.

Conflicts of Interest: We declare no conflicts of interest or financial interests. The Pacific University Institutional Review Board (IRB) has reviewed the work described in this study and it was given exemption status.

Treatment of Human Subjects: IRB exemption granted

\section{References}

1. Halbach JL, Sullivan LL. Teaching medical students about medical errors and patient safety: evaluation of a required curriculum. Acad Med 2005;80:600-606.

2. Kiersma ME, Darbishire, PL, Plake, KS, Oswald C, Walters BM. Laboratory Session to Improve First-year Pharmacy Students' Knowledge and Confidence Concerning the Prevention of Medication Errors. Am J Pharm Educ. 2009;73(6): Article 99.

3. Accreditation Council for Pharmacy Education. Accreditation standards and guidelines for the professional program in pharmacy leading to the doctor of pharmacy degree, Appendix 1. https://www.acpeaccredit.org/pdf/Standards2016FINAL.pdf Published 2016. Accessed June, 2017.

4. North American Pharmacist Licensure Examination. https://nabp.pharmacy/wpcontent/uploads/2017/09/NAPLEX-MPJE-BulletinAugust-2017.pdf, Accessed December 3, 2017.

5. Medina MS, Plaza CM, Stowe CD, et al. Center for the Advancement of Pharmacy Education 2013 educational outcomes. Am J Pharm Educ. 2013;77(8): Article162.

6. Haines ST, Pittenger AL, Stolte SK, et al. Core Entrustable Professional Activities for New Pharmacy Graduates. Am J Pharm Educ. 2017;81(1): Article S2.

7. Karimi R, Elbarbry F, and Fortner J. Integrative student learning: an effective team learning approach in a learner-centered paradigm. Inov Pharm. 2011;2(4):Article 57.

8. Fink LD. Creating Significant Learning Experiences. An Integrated Approach to Designing College Courses. San Francisco, CA: Jossey-Bass; 2003.

9. Bloom B. Taxonomy of educational objectives. The classification of educational goals, Handbook I Cognitive Domain. New York, NY: David McKay Company, Inc;1956.

10. Driscoll A and Wood S. Developing Outcomes-Based Assessment for Learner-Centered Education: A Faculty Introduction. Sterling, VA: Stylus Publishing, LLC;2007. 
Table 1. Questions used to generate the MESA report

What was the medication error and how did it happen?

What was the effect of the medication error to patients, healthcare providers, and the healthcare system at large?

Explain why the medication error happened and identify contributing factors (human and/or environmental factors) to the medication error.

How could patient (or patient's family) education prevent, or reduce the severity of, this medication error? How would you use the above information to establish a strategy in preventing a similar medication error?

Write two "Essential Keys" related to the medication error and the bridge between the medication error and biomedical and pharmaceutical sciences.

Table 2. Student perceptions of how the MESA activity assisted students in promoting their knowledge and skills in P1 curriculum. Assessment Question

$\%$ Strongly agree +

agree ( $\mathbf{t S D})$

MESA activity enhanced the awareness of devastating effects that a medication error can cause to patients, healthcare providers, and a healthcare community at large.

MESA activity promoted patient safety discussions to establish a plan to prevent a medication error.

$96.9(1.02)$

After working with your MESA project, you believe patient education plays an important role in the 97.9 (1.30) prevention of a medication error.

MESA activity increased the dynamic of student teamwork.

90.0 (2.60)

MESA activity integrated the knowledge and skills developed through the didactic part of the P1 92.3 (2.98) curriculum.

MESA activity promoted your critical thinking.

MESA activity promoted your active learning.

Encouraged you to be a self-directed learner.

Helped you to apply integrative pharmaceutical sciences to patient care.

MESA was an innovative student learning tool.

Fink's taxonomy: ${ }^{8}$ Fostered caring i.e., building new interests and values about something related to what you were learning. This helped you to be energized and engaged in the project.

Data are from three P1 cohorts: $n=70(72 \%) ; n=78(87 \%)$; and $n=77(79 \%)$.

Table 3. Student perception on the impact of MESA activity on Bloom's taxonomy educational objectives. ${ }^{9 \& 10}$

\begin{tabular}{|c|c|}
\hline Assessment Question & $\begin{array}{l}\text { \% Strongly agree + } \\
\text { agree ( } \pm \text { SD) }\end{array}$ \\
\hline $\begin{array}{l}\text { Promoted evaluation: the MESA activity created an environment where you gave defensible opinions and based } \\
\text { on given criteria you have judged accuracy, consistency, logic of information or argumentation. }\end{array}$ & $88.7(3.92)$ \\
\hline $\begin{array}{l}\text { Promoted synthesis: the MESA activity created an environment where you integrated and combined different } \\
\text { elements and parts to create a plan or structure that was not seen by you before. }\end{array}$ & $92.4(1.40)$ \\
\hline $\begin{array}{l}\text { Promoted analysis: the MESA activity created an environment where you broke the MESA project into its } \\
\text { constituent parts. You organized, clarified, concluded, or made inferences. This process of analysis helped you } \\
\text { understand the relationship among the constituent parts to grasp the big picture. }\end{array}$ & $94.6(1.55)$ \\
\hline $\begin{array}{l}\text { Promoted application: the MESA activity created an environment where you used and applied previously } \\
\text { learned knowledge from the P1 curriculum in new and concrete situations with a minimum of direction. }\end{array}$ & $94.6(1.53)$ \\
\hline $\begin{array}{l}\text { Promoted comprehension: the MESA activity created an environment where you comprehended information } \\
\text { based on prior learning from the P1 curriculum and translated knowledge into your own words. }\end{array}$ & $93.8(0.907)$ \\
\hline $\begin{array}{l}\text { Promoted knowledge: the MESA activity created an environment where you remembered or recognized } \\
\text { previously learned material from the P1 curriculum. }\end{array}$ & $93.7(2.35)$ \\
\hline
\end{tabular}

Data are from three P1 cohorts: $n=70$ (72\%); $n=78(87 \%)$; and $n=77(79 \%)$. 